\title{
Convergence Rates and Limit Theorems for the Dual Markov Branching Process
}

\author{
Anthony G. Pakes \\ School of Mathematics \& Statistics, University of Western Australia, 35 Stirling Highway, Crawley, WA 6009, Australia \\ Correspondence should be addressed to Anthony G. Pakes; tony.pakes@uwa.edu.au
}

Received 15 July 2016; Accepted 23 February 2017; Published 16 March 2017

Academic Editor: Ramón M. Rodríguez-Dagnino

Copyright ( 2017 Anthony G. Pakes. This is an open access article distributed under the Creative Commons Attribution License, which permits unrestricted use, distribution, and reproduction in any medium, provided the original work is properly cited.

\begin{abstract}
This paper studies aspects of the Siegmund dual of the Markov branching process. The principal results are optimal convergence rates of its transition function and limit theorems in the case that it is not positive recurrent. Additional discussion is given about specifications of the Markov branching process and its dual. The dualising Markov branching processes need not be regular or even conservative.
\end{abstract}

\section{Introduction}

The Markov branching process (MBP) is the discrete-state Markov process $\left(Z_{t}: t \geq 0\right)$ on the state-space $\mathcal{S}=\{0,1, \ldots\}$ whose transition function $\mathscr{F}(t)=\left[f_{i j}(t)\right]$ is standard and satisfies the branching property,

$$
f_{i j}(t)=\left(f_{1 j}(t)\right)^{* i}, \quad(i, j \in \mathcal{S}),
$$

where the asterisk denotes convolution. We allow the possibility that $\mathscr{F}(t)$ is dishonest, $\sum_{j \geq 0} f_{i j}(t)<1$. We shall see in the next section that $\mathscr{F}(t)$ is stochastically monotone; that is, $\sum_{k \geq j} f_{i k}(t)$ is nondecreasing in $i$, for each $j$. Consequently, as we explain in Section 3, it follows from the principal result (Siegmund's theorem) in [1] that the following definition of a dual MBP (DMBP) is not vacuous.

Definition 1. A DMBP $\left(X_{t}: t \geq 0\right)$ is an $\mathcal{S}$-valued Markov process whose transition function $P(t)=\left[p_{i j}(t)\right]$ satisfies

$$
\sum_{k \geq j} p_{i k}(t)=\sum_{k=0}^{i} f_{j k}(t), \quad(i, j, t \geq 0) .
$$

This process is the focus of the present paper. Definition 1 comes from [2] which also gives two equivalent definitions, one based on the $q$-matrix $Q=P^{\prime}(0)$ and one based on a so-called dual branching property ((22) below). We say a little about these definitions in Section 3. It is shown in [2] that $Q$ is regular and hence that $P(t)$ is minimal and honest. That paper also gives criteria for recurrence and transience. In particular the DMBP is positive recurrent if and only if the MBP is supercritical and the zero state is accessible, in which case the limiting-stationary law of the DMBP is geometric. A curious property is that $P(t)$ is strongly ergodic if and only if $\mathscr{F}(t)$ is dishonest.

There are two principal contributions of the present paper. The first is a study of the convergence properties of the transition function $P(t)$. If the zero state is inaccessible for the MBP, then it is absorbing and accessible for the DMBP. In this case there is a limiting conditional law (Theorem 3). In the positive recurrent case, Theorem 4 gives optimal convergence rates for the variation distance between $P(t)$ and its limiting geometric law. A by-product is an endogenous proof of the curious property mentioned above. Theorem 5 gives the exact rate at which $p_{i j}(t) \rightarrow 0$ in the case that the MBP is subcritical, and Theorems 6 and 8 deal with the critical case. These results follow from convergence rate results about $\mathscr{F}(t)$, the latter being fairly well known, at least when $\mathscr{F}(t)$ is honest.

The second contribution is an account of limit theorems for the DMBP when $\delta$ is not positive recurrent. Theorem 9 shows that if the MBP is critical or subcritical, then there is a family of constants $c_{t} \rightarrow \infty$ as $t \rightarrow \infty$ such that 
$\left(X_{t} / c_{t}\right)$ converges in law. The limit is a standard exponential law in the critical case and a finite mixture of Erlang laws in the subcritical case. In the subcritical case, convergence in law can be strengthened to almost sure convergence (Theorem 10). Theorems 11 and 12 are central limit analogues for this almost sure convergence. Theorems 9-11 are parallel to known results for the dual version of the simple branching process in [3]. The motivation for the model studied in that reference is the fact that the simple branching process in which immigration can occur is stochastically monotone; see (3) in [3]. Although it is not explicit in this reference, the model there is the Siegmund dual of a nonconservative or killed version of the simple branching process. This killed process is studied in [4]. The proof of almost sure convergence in Section 5 for the DMBP is quite different to that for the discrete-time version in [3]. That proof employs a general result about Markov chains for which no known continuoustime analogue is known. We remark that the dual MBP with immigration will be discussed in another paper.

Section 2 is devoted to consolidating the scattered literature concerning construction of the MBP and uniqueness of solutions of its Kolmogorov differential equation systems. In addition notation used in the sequel is established. In Section 3 we supplement the discussion of the definitions of the DMBP in [2]. In particular, Proposition 2 gives a very direct proof that $P(t)$ is a minimal transition function. This is in contrast to the observation in [2] that this result follows from a more specialised criterion in [5].

The presentation in this paper stresses the relation with branching processes, whereas that in [2] is closer to the analytical approach used more commonly in literature on Markov construction theory. Our approach results in a more intrinsic development and, we believe, the various results have greater intuitive appeal when expressed in terms used in the branching process literature.

\section{Defining and Constructing the MBP}

Perusing the standard monograph accounts of branching processes reveals a variety of definitions of the MBP, all of which are motivated by the idea of the MBP as a model of a population of reproducing individuals. The branching property (1) expresses the notion of independence of separate lines of descent, and we begin by summarizing its consequences. Define the probability generating functions $F_{i}(s, t)=\sum_{j \geq 0} f_{i j}(t) s^{j}$, and let $F(s, t)=F_{1}(s, t)$. The branching property is equivalent to the relation $F_{i}(s, t)=(F(s, t))^{i}$. This identity implies that state 0 is absorbing. If $0<s<1$ then

$$
f_{i j}(t) \leq s^{-j}(F(s, t))^{i} \longrightarrow 0, \quad(i \longrightarrow \infty)
$$

that is, $\mathscr{F}(t)$ is Feller. This property has the important consequence that all states are stable, meaning that the jump rates $\widetilde{q}_{i j}:=f_{i j}^{\prime}(0)$ are all finite; see p. 43 in [6] for the definition and its consequences. Let $\rho=-\widetilde{q}_{11}$, assumed positive, and let $p_{j}=\tilde{q}_{1 j} / \rho$ for $j \neq 1$, and $p_{1}=0$. It follows from (1) that $q$-matrix $\widetilde{Q}=\left[\widetilde{q}_{i j}\right]$ is specified by

$$
\widetilde{q}_{i j}= \begin{cases}\rho i p_{j-i+1} & \text { if } i, j=0,1, \ldots, i \neq j, \\ -\rho i & \text { if } i=j=0,1, \ldots\end{cases}
$$

Assume $p_{0}<1$ and $0 \leq \sum_{j \geq 0} p_{j}=1-d$, where $0 \leq d \leq 1$, and $d=0$ if and only if $\widetilde{Q}$ is conservative. Let $f(s)=\sum_{j \geq 0} p_{j} s^{j}$ and $a(s)=(f(s)-s) \rho$. We need the following notation for the sequel. Let $m=f^{\prime}(1)(\leq \infty)$ if $d=0$ and $m=\infty$ if $d>0$. If $q$ denotes the least nonnegative solution of $f(s)=s$, then $q=1$ if $m \leq 1, q<1$ if $m>1$, and $q=0$ if and only if $p_{0}=0$ (which implies that $m>1$ ).

Often the form (4) of $q$-matrix is chosen to model a population process where individuals live and reproduce independently with $\exp (\rho)$ lifetime law and, at the time of death, they are replaced by $j$ individuals with probability $p_{j}$ $(j=0,2, \ldots)$ or by infinitely many with probability $d$. Thus $m$ is the mean per capita number of offspring, and we delineate the subcritical, critical, and super-critical cases, according to $m<1, m=1$, or $m>1$, respectively. Then $Z_{t}$ is the population size at time $t$. If $d>0$ then the state-space is extended to $\mathcal{S} \cup\{\infty\}$, where $\infty$ is an absorbing state. In this case sample paths either hit the zero state or hit $\infty$ via a single infinite jump at the first occurrence of an infinite litter. In all cases, the former occurs with probability $q^{i}$ if $Z_{0}=i$.

The population image is captured by the minimal transition function corresponding to $\widetilde{Q}$. This satisfies the backward and the forward Kolmogorov equations. General theory shows that $\mathscr{F}(t)$ is minimal because it possesses the Feller property; see p. 81 in [6]. We show this below without appealing to the Feller property. The forward equation system can be wrapped up into the single linear first-order partial differential equation:

$$
\frac{\partial \Phi_{i}(s, t)}{\partial t}=a(s) \frac{\partial \Phi_{i}(s, t)}{\partial s}, \quad \Phi(s, 0)=s^{i},
$$

where $\Phi_{i}(s, t)(i=1,2, \ldots)$ represents the probability generating functions for any $\widetilde{Q}$-function. This equation has a unique solution in the class of functions which are holomorphic in the open unit disc; see p. 119 in [7]. The proof in this reference is based on a general uniqueness theorem for linear partial differential equations and although it assumes that $\widetilde{Q}$ is conservative, the proof is valid in general. Alternatively, a unique solution of the forward system within the class of transition functions follows from Reuter's criterion that such uniqueness is equivalent to the assertion that the only sequence $\left\{v_{j}: j \in \mathcal{S}\right\} \in \ell_{1}^{+}$such that

$$
\lambda v_{j}=\sum_{i \geq 0} v_{i} \widetilde{q}_{i j}, \quad(j \geq 0)
$$

for some (and hence all) $\lambda>0$ is $v_{j} \equiv 0$. The explicit form of this equation is

$$
(\lambda+\rho j) v_{j}=\rho \sum_{i=0}^{j+1} i v_{i} p_{j-i+1} .
$$


If $p_{0}=0$ then setting $j=0$ shows that $\lambda v_{0}=0$, so by recursion, $v_{j} \equiv 0$. If $p_{0}>0$ it is clear that, given a value of $v_{0}$, this system can be solved recursively for $v_{1}, v_{2}, \ldots$. So a nonnegative solution certainly exists. Estimates showing that $\sum_{j} v_{j}=\infty$ are derived in [8].

The same end is achieved in [6] (p. 114) using a generating function approach. The proof there rather obscures the fact that (7) does have a solution. A tidier version is to formally define the generating function $N(s)=\sum_{j \geq 0} v_{j} s^{j}$ and observe that (7) yields the differential equation $a(s) N^{\prime}(s)=\lambda N(s)$ with solution

$$
N(s)=v_{0} \exp \left(\lambda \int_{0}^{s} \frac{d u}{a(u)}\right) .
$$

If $p_{0}>0$ and $s<q$ then the integrand has a power series expansion with nonnegative coefficients. It follows that $N(s)$ is holomorphic in the disc $|s|<q$ and, hence, if $\nu_{0}>0$, its coefficients solve (7). However, $N\left(q^{-}\right)=\infty$, so $\left\{v_{i}\right\} \notin \ell_{1}^{+}$.

Consequently, the minimal $\widetilde{Q}$-function is the only transition function solution of the forward system. But if (5) holds with $i=1$, then it is solved for general $i$ by $\left(\Phi_{1}(s, t)\right)^{i}$. It follows immediately from Harris' version of the uniqueness theorem that this can be the only solution holomorphic in the unit disc, and hence the minimal $\widetilde{Q}$-function has the branching property.

It does not seem possible to so directly draw this conclusion from the uniqueness given by Reuter's criterion. However, the branching property implies that the ChapmanKolmogorov equations for $\mathscr{F}(t)$ can be rendered as the functional equation $F(s, t+u)=F(F(s, u), t)$ and hence that

$$
\begin{aligned}
\frac{\partial}{\partial t} F(s, t+u) & =\frac{\partial}{\partial u} F(s, t+u) \\
& =\frac{\partial F(s, u)}{\partial u} F^{\prime}(F(s, u), t),
\end{aligned}
$$

where the prime denotes partial differentiation with respect to $s$. Setting $u=0$, it follows from (4) that the first factor on the right-hand side is $a(s)$, and hence $F(s, t)$ solves (5) with $s<1$. It follows that $\mathscr{F}(t)$ solves the forward system, and we conclude that $\mathscr{F}(t)$ is the minimal transition function.

The backward system is uniquely satisfied by $\mathscr{F}(t)$ if it is honest. If $\widetilde{Q}$ is conservative and $\mathscr{F}(t)$ is dishonest, then the backward system has uncountably many transition function solutions. The single entrance solutions are investigated in [9]. Of course, none of these has the branching property.

Surprisingly, if $\widetilde{Q}$ is not conservative then the backward system is uniquely solved by the minimal transition function. This follows from another Reuter criterion which asserts that uniqueness within the class of $\widetilde{Q}$-functions is equivalent to the condition that the only solution $\{x(i): i \in \mathcal{S}\} \in \ell_{\infty}^{+}$of the system

$$
\lambda x(i)=\sum_{j \geq 0} \tilde{q}_{i j} x(j), \quad(i \geq 0)
$$

for some (and hence all) $\lambda>0$ is $x_{i} \equiv 0$. This criterion is shown to hold in [8] (pp. 224-226). The following proof is simpler, though preserving the spirit of [8].
So, assume that there is a nontrivial solution $\{x(i)\}$, as above. Note first that $x(0)=0$ because $\widetilde{q}_{0 j} \equiv 0$. If $i \geq 1$, then the explicit form of the above system can be written as

$$
\left(1+\frac{\lambda}{\rho i}\right) x(i)=\sum_{j \geq i-1} p_{j-i+1} x(j), \quad(i \geq 0) .
$$

Suppose there exists $i^{\prime} \geq 1$ such that $x\left(i^{\prime}\right) \geq x(i)$ for all $i$. Setting $i=i^{\prime}$ yields $\left(1+\lambda / \rho i^{\prime}\right) x\left(i^{\prime}\right) \leq x\left(i^{\prime}\right)$, a contradiction. It follows that there is a strictly increasing sequence of positive integers $\left\{i_{n}: n \geq 1\right\}$ such that, for all $n, x\left(i_{n}\right)<x\left(i_{n+1}\right)$ and $x\left(i_{n}\right) \geq \max _{i \leq i_{n}} x(i)$. Consequently $x(i) \leq \bar{x}:=\lim _{n \rightarrow \infty} x\left(i_{n}\right)=$ $\sup _{i \geq 1} x(i)$, and hence

$$
\left(1+\frac{\lambda}{\rho i_{n}}\right) x\left(i_{n}\right) \leq \bar{x} \sum_{j \geq i-1} p_{j-i+1}=\bar{x}(1-d) .
$$

Letting $n \rightarrow \infty$ yields $\bar{x} \leq \bar{x}(1-d)$, implying that $\bar{x}=0$, a contradiction, if $d>0$. Hence the only bounded solution is $x(i) \equiv 0$.

Although $\mathscr{F}(t)$ is the unique solution of the backward and the forward systems if $\widetilde{Q}$ is not conservative, it is not the unique $\widetilde{Q}$-function. Uniqueness in this case is equivalent to two conditions. The first is the above Reuter condition for uniqueness of the forward system, and the second is that, for any $\lambda>0$, there is a constant $K_{\lambda}>0$ such that the inequality

$$
\lambda \int_{0}^{\infty}\left(\sum_{j \geq 0} f_{i j}(t)\right) e^{-\lambda t} d t \geq K_{\lambda}
$$

holds for all $i$; see p. 150 in [6]. However, the sum equals $(\delta(t))^{i}$ where we define $\delta(t)=F(1, t)$, and since $\delta(t)<1$, we see that the left-hand side tends to zero as $i \rightarrow \infty$. Thus the criterion for uniqueness is violated, meaning there exists a $\widetilde{Q}$-function which is not a solution of either the backward or forward systems.

The MBP is defined in [7] to be a Markov process whose transition function solves the forward system obtained from $\widetilde{Q}$. The branching property is derived from this (Harris) definition. The MBP definition we adopt in Section 1 is given in [10] but not used there. Instead, (4) is motivated from population growth considerations and then used in a description of the Feller construction of the minimal process. The specification in [11] proceeds from a construction involving Galton-Watson trees with randomized split times. Also described in [11] is the Athreya-Karlin construction [12] which, in essence, is the Markov process whose jump chain is a left-continuous random walk and holding times in state $i$ have the exponential $\exp (\rho i)$ law. This construction is described too in [10]. The monograph [8], on Markov construction theory, uses our definition. This is the only treatment which, to our knowledge, gives any attention to the nonconservative case. Finally, we mention that, in [13] (see Section 3 there), the author clearly distinguishes between what he calls the probabilistic definition, that is, a Markov process having the branching property, and the analytic definition, that is, the above Harris definition. The proof in [13] of the equivalence of these definitions uses facts already 
mentioned that the branching property implies the Feller property, and this implies minimality.

The backward system for the minimal $\widetilde{Q}$-function is expressed in terms of the probability generating function $F(s, t)$ as

$$
\frac{\partial F(s, t)}{\partial t}=a(F(s, t)), \quad(0 \leq s \leq 1),
$$

or as

$$
\int_{s}^{F(s, t)} \frac{d u}{a(u)}=t
$$

in its integrated form. The total mass function $\delta(t):=P_{1}\left(Z_{t}<\right.$ $\infty)=F(1, t)$ satisfies

$$
\int_{\delta(t)}^{1} \frac{d s}{s-f(s)}=\rho t .
$$

This implies the known criterion that $\mathscr{F}(t)$ is honest if and only if

$$
C:=\int_{s^{\prime}}^{1} \frac{d s}{s-f(s)}=\infty,
$$

where $q<s^{\prime}<1$. This appears in [7] where it is attributed to E. B. Dynkin. See the introduction of [14] for remarks on these attributions. Note that $C<\infty$ only if $m>1$ and certainly if $\widetilde{Q}$ is not conservative. It follows from (16) that if $C<\infty$ then $q<\delta(t)<1$, and $\delta(t) \downarrow q$ as $t \uparrow \infty$.

Suppose that $\widetilde{Q}$ is not regular. It is not at first apparent why the constructions of transition functions in [9] need $\widetilde{Q}$ to be conservative. The transition functions considered in that paper (see (2.11) there) have the form of the sum of $f_{i j}(t)$ plus a convolution integral. A computation shows that the derivative of this added term is proportional to $\delta^{\prime}(0)$. Since $\delta(0)=1$, we see from (14) with $s=1$ that $\delta^{\prime}(0)=a(1)=-\rho d$, and hence the transition functions constructed in [9] are $\widetilde{Q}$ functions only if $\widetilde{Q}$ is conservative. On the other hand, the limiting conditional theorem in [9] (p. 743) holds for the nonconservative MBP. Finally, we mention that several of the results in [15] for the nonconservative linear birth and death process carry over to the MBP.

\section{On Definitions of the Dual MBP}

As we mentioned in the introduction, three equivalent definitions of the DMBP are presented in [2]. Definition (i) is just Definition 1 above. Definition (ii) asserts that the DMBP is the Markov process whose $q$-matrix has the form

$$
q_{i j}= \begin{cases}(j+1) a_{i-j}-j a_{i-j+1}, & \text { if } i \geq j-1, \\ 0, & \text { if } i<j-1,\end{cases}
$$

where $\left\{a_{j}: j \geq-1\right\}$ is a sequence satisfying

$$
\begin{aligned}
a_{-1} & =0, \\
a_{0} & \leq 0, \\
a_{1} & \geq a_{2} \geq \cdots \geq 0, \\
a_{0}+a_{1} & >0 .
\end{aligned}
$$

In particular $q_{i i}=a_{0}-\left(a_{1}-a_{0}\right) i$. So $a_{1} \geq a_{0}$, and if equality holds, then $a_{0}=0$. Hence we always assume that $a_{1}-a_{0}>0$ to avoid a trivial situation. In this case the parameters $\left\{a_{j}\right\}$ and $\left\{\rho ; p_{j}\right\}$ are related through

$$
\begin{aligned}
& a_{0}=-\rho p_{0}, \\
& a_{j}=\rho\left(1-\sum_{r=0}^{j} p_{r}\right), \quad(j \geq 1) .
\end{aligned}
$$

Note that $\lim _{j \rightarrow \infty} a_{j}=0$ if and only if $\widetilde{Q}$ is conservative. In addition $a_{1}-a_{0}=\rho$, and the inverse relation is

$$
\begin{aligned}
\rho & =a_{1}-a_{0}, \\
p_{0} & =-\frac{a_{0}}{\rho}, \\
p_{1} & =0, \\
p_{j} & =\frac{\left(a_{j-1}-a_{j}\right)}{\rho}, \quad(j \geq 1),
\end{aligned}
$$

implying that $p_{0}=1$ unless there is at least one strict inequality in the chain (19).

Definition (iii) is that the DMBP is a Markov process whose transition function has the dual branching property

$$
p_{i j}(t)=\sum_{r=0}^{i} p_{i-r, j-1}(t)\left(p_{r-1,0}(t)-p_{r 0}(t)\right),
$$

$$
(i \geq 0, j \geq 1)
$$

and $p_{-1,0}(t) \equiv 1$. The proof in [2] is a mostly analytic demonstration that (ii) $\Rightarrow$ (i) $\Rightarrow$ (iii) $\Rightarrow$ (ii). The following proof that (i) $\Leftrightarrow$ (iii) supplements the treatment in that reference.

We note first that, in the case that $\widetilde{Q}$ is conservative, the paper [16] gives an analytic proof that $\mathscr{F}(t)$ is stochastically monotone. A direct and completely general demonstration follows by expressing (1) as

$$
f_{i j}(t)=P\left(Z_{t} \mid Z_{0}=i\right)=P\left(Z_{1, t}+\cdots+Z_{i, t}=j\right),
$$

where the processes $\left\{\left(Z_{r, t}\right): r=1,2, \ldots\right\}$ are independent copies of $\left(Z_{t}\right)$ started with $Z_{0}=1$. It follows that

$$
P\left(Z_{t} \geq j \mid Z_{0}=i\right)=P\left(Z_{1, t}+\cdots+Z_{i, t} \geq j\right),
$$

and since the summands are nonnegative it is evident that $\mathscr{F}(t)$ is SM. This is valid whether or not $\widetilde{Q}$ is regular, not regular, or not even conservative. Hence Siegmund's theorem is applicable in each of these circumstances because, if $\mathscr{F}(t)$ is dishonest, then the added boundary state $\infty$ is absorbing. Hence there is a Markov process $\left(X_{t}\right)$ satisfying Definition (i).

Next, define the generating function $G_{j}(s, t)=$ $\sum_{i \geq 0} s^{i} p_{i j}(t)$, which is finite if $|s|<1$. The relation (2) is equivalent to the identity

$$
\sum_{k \geq j} G_{k}(s, t)=\frac{(F(s, t))^{j}}{1-s}
$$


from which we obtain the fundamental identity

$$
G_{j}(s, t)=\frac{1-F(s, t)}{1-s}(F(s, t))^{j}, \quad(j \geq 0) .
$$

Summing over all $j$ yields $\sum_{i \geq 0} s^{i} P_{i}\left(X_{t}<\infty\right)=(1-s)^{-1}$. This implies that $P_{i}\left(X_{t}<\infty\right) \equiv 1$; that is, $P(t)$ is always an honest transition function. We show below that it is a minimal transition function.

We now show that (1) implies the identity (22). If $j \geq 1$ then (26) implies that

$$
\begin{aligned}
G_{j}(s, t) & =G_{j-1}(s, t) F(s, t) \\
& =G_{j-1}(s, t)\left[1-(1-s) G_{0}(s, t)\right] .
\end{aligned}
$$

Equating the coefficients of $s^{i}$ on each side of this identity together with a little manipulation yields (22).

In the opposite direction, suppose that we start with Definition (iii). Then defining $G_{j}(s, t)$ as above and $F(s, t)=$ $1-(1-s) G_{0}(s, t)$ we see that $(22)$ is equivalent to (26). In addition,

$$
F(s, t)=1-p_{00}(t)+\sum_{r \geq 0} s^{r}\left(p_{r-1,0}(t)-p_{r 0}(t)\right) .
$$

But (22) implies that the coefficient of $s^{r}$ is nonnegative and also that $F(1-, t)=1-\lim _{r \rightarrow \infty} p_{r 0}(t) \leq 1$. Hence $F(s, t)$ is the probability generating function of a possibly defective discrete law. It follows from this that $(F(s, t))^{j}$ has a power series expansion $\sum_{i \geq 0} f_{j i}(t) s^{i}$ and that the coefficients possess the branching property (1), after swapping $i$ and $j$. Since $P(t)$ is standard, $G_{0}(s, 0+)=1$ and hence $F(s, 0+)=s$. It follows that $f_{i j}(0+)=\delta_{i j}$.

It remains to show that $f_{i j}(t)$ satisfy the ChapmanKolmogorov equations. Since we have established the branching property, this will follow once we demonstrate the composition identity

$$
F(F(s, t), u)=F(s, t+u) .
$$

To prove this, observe that the Chapman-Kolmogorov equations for $P(t)$ yield for $j \geq 0$,

$$
\begin{aligned}
G_{j} & (s, t+u)=G_{0}(s, t)(F(s, t+u))^{j} \\
& =\sum_{k \geq 0} G_{k}(s, t) p_{k j}(u) \\
& =G_{0}(s, t) \sum_{k \geq 0}(F(s, t))^{k} p_{k j}(u) \\
& =G_{0}(s, t) G_{j}(F(s, t), u) \\
& =G_{0}(s, t) G_{0}(F(s, t), u)[F(F(s, t), u)]^{j} .
\end{aligned}
$$

Setting $j=0$ yields

$$
G_{0}(s, t+u)=G_{0}(s, t) G_{0}(F(s, t), u),
$$

and (29) follows from the definition of $G_{0}(s, t)$.
It follows that $f_{i j}(t)$ we have constructed comprise a standard transition function $\mathscr{F}(t)$ which possesses the branching property. Hence, as we have seen, it is a minimal transition function. Consequently there is a MBP whose transition function is that which we have constructed and that it is linked to $P(t)$ via (18). But this identity is equivalent to (2), and hence Definitions (i) and (iii) are equivalent.

We have seen that the transition function $P(t)$ is always honest. A sufficient condition for it to be minimal is the Feller property $\lambda_{j}(t):=\lim _{i \rightarrow \infty} p_{i j}(t)=0$ for all $j, t \geq 0$ (p. 43 in [6]). To check this property, note that (2) implies that $P(t)$ is $\mathrm{SM}$, and this implies the existence of the limit $\lambda_{j}(t)$. This limit is evaluated by applying an Abelian theorem for power series to (18) to obtain

$$
\lambda_{j}(t)=\lim _{s \rightarrow 1-}(1-s) G_{j}(s, t)=(1-\delta(t))(\delta(t))^{j}
$$

and this is zero if and only if $\delta(t) \equiv 1$, that is, if and only if $\mathscr{F}(t)$ is honest.

The fact that $P(t)$ is always minimal is a corollary of a simple general result which we state as follows.

Proposition 2. Suppose $\mathscr{F}(t)$ is any $S M$ transition function whose q-matrix elements $\tilde{q}_{i j}$ satisfy $\widetilde{q}_{0 i} \equiv 0$ and which admits a dual transition function $P(t)$ in the sense of (2).

(1) The dual q-matrix $Q$ is specified by

$$
q_{i j}=\sum_{k=0}^{i}\left(\tilde{q}_{j k}-\tilde{q}_{j+1, k}\right),
$$

and $Q$ is conservative.

(2) Suppose that there is an integer $b \geq 1$ such that $\tilde{q}_{i j}=0$ if $i-j>b$, and there exists $i^{\prime} \in \mathbb{N}$ such that $q(i):=-q_{i i}$ is nondecreasing for $i \geq i^{\prime}$. Then $Q$ is regular if

$$
\sum_{n>i^{\prime}} \frac{1}{q(i+n b)}=\infty, \quad \forall i \geq 0 .
$$

Proof. Since $Q=P^{\prime}(0)$, (33) follows by differentiation. Summing over $j$ yields $\sum_{j \geq 0} q_{i j}=\sum_{k=0}^{i} \widetilde{q}_{0 k}=0$ for all $i$, proving Assertion (1). For (2), it follows from the bounded increment assumption and (33) that if $j-i>b$, then $\widetilde{q}_{j k}=$ $\tilde{q}_{j+1, k}=0$ if $k \leq i$, implying that $q_{i j}=0$. So if $\left(J_{n}\right)$ denotes the jump chain of $\left(X_{t}\right)$, then $J_{n+1}-J_{n} \leq b$ almost surely. Hence $J_{n} \leq i+n b$, implying that $1 / q\left(J_{n}\right) \geq 1 / q(i+n b)$ for all sufficiently large $n$. Thus (34) implies that $\sum_{n \geq 1} 1 / q\left(J_{n}\right)=$ $\infty$, almost surely. It follows from the (generalized) FellerLundberg criterion (e.g., p. 337 in [17]) that the Feller minimal process constructed from $Q$ is honest, and hence its transition function coincides with $P(t)$. Thus $Q$ is regular.

The jump chain of the MBP is a random walk which is skip-free to the left, and hence we can take $b=1$ in Assertion (2) of Proposition 2. In addition, it follows from (33) and (4) that

$$
q(i)=-\tilde{q}_{i i}+\tilde{q}_{i+1, i}-\tilde{q}_{i, i-1}=i \rho+p_{0},
$$


and hence (34) is satisfied. It follows that the DMBP transition function is honest and minimal. Some manipulation with (4) and (33) will show that $\widetilde{Q}$ has the form (19).

At this point we mention two extreme cases. If $p_{0}=1$ then the generating MBP is the linear death process and it is easy to show from (18) that

$$
\sum_{j \geq 0} p_{i j}(t) z^{j}=\frac{e^{-\rho t}}{1-z\left(1-e^{-\rho t}\right)}\left(\frac{z}{1-z\left(1-e^{-\rho t}\right)}\right)^{i} .
$$

It follows that the DMBP is a linear birth process with an independent immigration component in which individuals arrive at the event times of a Poisson process having rate $\rho$.

If $d=1$ and $Z_{0}=i \geq 1$, then the sample path has a single jump to $\infty$ at a time which has the $\exp (\rho i)$ law. In terms of the population model, the first reproduction event results in an infinite number of offspring. The corresponding DMBP can be regarded as a uniform catastrophe process: if $X_{t}=i \geq 1$, then the next jump occurs after a time having the $\exp (\rho i)$ law, and it is into a state $j=0, \ldots, i-1$ with probability $i^{-1}$. The zero state is absorbing.

\section{Long-Term Behaviour of $P(t)$}

Recall that $F(t):=F(0, t)$ is the distribution function of the hitting time of state 0 by the MBP when $Z_{0}=1$ and that $\lim _{t \rightarrow \infty} F(t)=q$. It follows from (26) that

$$
p_{0 j}(t)=(1-F(t))(F(t))^{j}, \quad(j \geq 0)
$$

and hence that the DMBP is positive recurrent if and only if $p_{0}>0$ and $m>1$. In this case there is a geometric limitingstationary law $\lim _{t \rightarrow \infty} p_{i j}(t)=\pi_{j}=(1-q) q^{j}$. This is an alternative approach to Theorem 3.1 in [2].

The next two theorems require the following extension of a known result (p. 115 in [10]). Still assuming that $m>1$, we define the parameter $\beta=\left(1-f^{\prime}(q)\right) \rho$. Then

$$
\lim _{t \rightarrow \infty} e^{\beta t}[F(s, t)-q]=U(s)
$$

where $U(s)=\sum_{j \geq 0} u_{j} s^{j}, u_{0}<0$ if $p_{0}>0$ and $u_{0}=0$; otherwise, $u_{j} \geq 0$ if $j \geq 1$ and $U(q)=0$. Recalling (17), we see that $U(1)=\infty$ if $C=\infty$ and $U(1)<\infty$ if $C<\infty$.

It follows from (18) and (20) that $q_{i, i+1}=(i+1) \rho p_{0}$. Assume for the following that $p_{0}=0$ in which case sample paths of the DMBP are nonincreasing step functions and 0 is an absorbing state. Thus the DMBP is a kind of generalized death process, consistent with the fact that the pure (resp., linear) death process is the Siegmund dual of the pure (resp., linear) birth process. This fact follows by inspection of $q$ matrices exhibited in Example (d) of [1]. Still assuming $p_{0}=$ 0 , it follows from (38) that

$$
\lim _{t \rightarrow \infty} e^{i \rho t} f_{i j}(t)=u_{i j}, \quad(i, j \geq 1)
$$

where $\sum_{j \geq 1} u_{i j} s^{j}=(U(s))^{i}$ and $u_{i j}=0$ if $i>j$.

Let $T$ be the hitting time of 0 by $\left(X_{t}\right)$. Since 0 is accessible from all $i \geq 1$, it follows that $P_{i}(T<\infty)=1$.
Theorem 3. If $p_{0}=0$ then

$$
\begin{aligned}
\lim _{t \rightarrow \infty} e^{j \rho t} p_{i j}(t) & =\sum_{k=j}^{i} u_{j k} \quad(0 \leq j \leq i), \\
\lim _{t \rightarrow \infty} P_{i}\left(X_{t}=j \mid T>t\right) & =\delta_{1 j} .
\end{aligned}
$$

Proof. It follows from (39) that $f_{j+1, k}(t)=o\left(f_{j k}(t)\right)$, and hence (2) yields

$$
\begin{aligned}
p_{i j}(t)=\sum_{k=0}^{i}\left(f_{j k}(t)-f_{j+1, k}(t)\right) \sim \sum_{k=0}^{i} f_{j k}(t), & \\
& (t \longrightarrow \infty),
\end{aligned}
$$

and (40) follows from (39). Next, $f_{00}(t) \equiv 1$ and hence $p_{i 0}(t)=1-\sum_{k=1}^{i} f_{1 k}(t)$, and it follows from (40) that

$$
e^{\rho t} P_{i}(T>t) \longrightarrow \sum_{k=1}^{i} u_{1 k}
$$

Assertion (41) follows from this estimate and (40).

Further calculation shows that the speed of convergence in (41) is characterized by

$$
e^{\rho t} \sum_{j \geq 1}\left|P_{i}\left(X_{t}=j \mid T>t\right)-\delta_{1 j}\right| \longrightarrow \frac{2 \sum_{k=2}^{i} u_{2, k}}{\sum_{k=1}^{i} u_{k}} .
$$

Next we let $p_{0}>0$ and determine the rate at which the variation distance between $P(t)$ and the geometric limitingstationary law approaches zero. This is equivalent to estimating the speed at which $v_{i}(t):=\sum_{j \geq 0}\left|p_{i j}(t)-\pi_{j}\right| \rightarrow 0$. A more stringent measure of the speed of convergence is to require that this limit relation holds uniformly in $i$, that is, that $v(t):=\sup _{i>0} v_{i}(t) \rightarrow 0$. If this holds then $P(t)$ is said to be strongly ergodic. As observed in [2], $P(t)$ is strongly ergodic if and only if $\mathscr{F}(t)$ is dishonest. This follows from Theorem 2.2 in [18] asserting that an ergodic and SM transition function is strongly ergodic if and only if it is not Feller, and we have seen above that $P(t)$ is Feller if and only if $\mathscr{F}(t)$ is honest.

The following result gives the rate of convergence to zero of $v_{i}(t)$ and $v(t)$. As a by-product, it gives a proof of the result just discussed without using Theorem 2.2 in [18].

Theorem 4. Suppose $p_{0}>0$ and $m>1$. (a) For all $i \geq 0$, $v_{i}(t)=O\left(e^{-\beta t}\right)$.

(b) Suppose also that $C<\infty$. Then $v(t)=O\left(e^{-\beta t}\right)$.

These convergence rate estimates are optimal.

Proof. We obtain exact element-wise convergence rates as follows. It follows from (26) that

$$
\begin{aligned}
G_{j}(s, t)-\frac{\pi_{j}}{1-s} \\
=\frac{(1-F(s, t))\left[(F(s, t))^{j}-q^{j}\right]-(F(s, t)-q) q^{j}}{1-s},
\end{aligned}
$$


and hence (38) yields

$$
\begin{aligned}
& \lim _{t \rightarrow \infty} e^{\beta t}\left(G_{j}(s, t)-\frac{\pi_{j}}{1-s}\right) \\
& =\left(j q^{j-1}-(j+1) q^{j}\right) \frac{U(s)}{1-s} .
\end{aligned}
$$

This implies that

$$
\begin{aligned}
\ell_{i j} & :=\lim _{t \rightarrow \infty} e^{\beta t}\left[p_{i j}(t)-\pi_{j}\right] \\
& =\left(\sum_{k=0}^{i} u_{k}\right)\left(j q^{j-1}-(j+1) q^{j}\right) .
\end{aligned}
$$

Note that $\ell_{00}>0$.

Next, (26) can be expressed as

$$
G_{j}(s, t)=G_{0}(s, t) \sum_{k \geq 0} f_{j k}(t) s^{k},
$$

from which we obtain the representation

$$
p_{i j}(t)=\sum_{k=0}^{i} p_{i-k, 0}(t) f_{j k}(t)=r_{i j}(t)+R_{i j}(t),
$$

where $r_{i j}(t)=p_{i 0}(t)(F(t))^{j}$ and

$$
R_{i j}(t)=\sum_{k=1}^{i} p_{i-k, 0}(t) f_{j k}(t) .
$$

Observing that $r_{i j}(t) \rightarrow \pi_{j}$, we write

$$
\begin{aligned}
r_{i j}(t)-\pi_{j}= & \left(p_{i 0}(t)-\pi_{0}\right)(F(t))^{j} \\
& -\pi_{0}\left(q^{j}-(F(t))^{j}\right),
\end{aligned}
$$

and hence

$$
\begin{aligned}
\sum_{j \geq 0}\left|r_{i j}(t)-\pi_{j}\right| & \leq \max \left[\frac{p_{i 0}(t)-\pi_{0}}{1-F(t)}, \frac{q-F(t)}{1-F(t)}\right] \\
& =O\left(e^{-\beta t}\right),
\end{aligned}
$$

by virtue of (38) and (47).

Next, (2) implies that

$$
p_{i 0}(t)=\sum_{k>i} f_{1 k}(t)=P_{1}\left(i<Z_{t}<\infty\right) \leq 1-F(t)
$$

and, hence, if $s \in(0,1)$, then

$$
\begin{aligned}
0 & \leq R_{i j}(t) \leq(1-F(t)) \sum_{k=1}^{i} f_{j k}(t) \\
& <(1-F(t)) s^{-i} \sum_{k=1}^{i} f_{j k}(t) s^{k} \\
& <(1-F(t)) s^{-i}\left((F(s, t))^{j}-(F(t))^{j}\right) .
\end{aligned}
$$

Summing over $j$, it follows from (49) and (52) that

$$
v_{i}(t) \leq O\left(e^{-\beta t}\right)+s^{-i} \frac{F(s, t)-F(t)}{1-F(s, t)} .
$$

But (38) implies that $e^{\beta t}[F(s, t)-F(t)] \rightarrow U(s)-u_{0}$, thus proving Assertion (a). It follows from (47) that the exponent $\beta$ cannot be increased.

Returning to (53), observe that

$$
p_{i 0}(t)-\pi_{0}=q-F(t)-\sum_{k=1}^{i} f_{1 k}(t) .
$$

The sum is zero if $i=0$, so the right-hand side decreases as $i$ increases. Consequently

$$
\sup _{i \geq 0}\left|p_{i 0}(t)-\pi_{0}\right|=\max [q-F(t), \delta(t)-q],
$$

and we see that the right-hand side tends to zero if and only if $\delta(t)<1$, that is, if and only if $C<\infty$. Hence $P(t)$ is not strongly ergodic if $C=\infty$.

Suppose that $C<\infty$. Then (38) with $s=0$ and $s=1$ shows that $\sup _{i \geq 0}\left|p_{i 0}(t)-\pi_{0}\right|=O\left(e^{-\beta t}\right)$. Using this estimate with (52) yields

$$
\sup _{i \geq 0} \sum_{j \geq 0}\left|r_{i j}(t)-\pi_{j}\right|=O\left(e^{-\beta t}\right) \text {. }
$$

In a similar way, it follows from (50) that

$$
\begin{aligned}
R_{i j}(t) & \leq(1-F(t)) \sum_{k \geq 1} f_{j k}(t) \\
& =(1-F(t))\left((\delta(t))^{j}-(F(t))^{j}\right),
\end{aligned}
$$

and hence $\sup _{i \geq 0} R_{i j}(t)=O\left(e^{-\beta t}\right)$. It follows that $P(t)$ is strongly ergodic if $C<\infty$, and Assertion (2) follows from these estimates.

We now consider the case $m \leq 1$ in which case $p_{0}>0$. So setting $j=0$ in (26) yields $p_{i 0}(t)=\sum_{k>i} f_{i k}(t)>0$ for all $i$, and hence 0 is accessible from $\mathbb{N}$. In addition $q_{i, i+1}>0$ for all $i$, and hence $\delta$ is irreducible. Taking $s=0$ in (26) yields $p_{00}(t)=1-F(t) \rightarrow 0$, since $F(t) \rightarrow 1$, so $\mathcal{S}$ is not positive recurrent. It follows that $\mathcal{S}$ is transient if the mean time to extinction of the MBP $\mu_{e}=\int_{0}^{\infty}(1-F(t)) d t<\infty$ and nullrecurrent if $\mu_{e}=\infty$. Let $s=F(t)$ in the integral and observe that (14) implies that $d t=d s /(f(s)-s) \rho$. It follows that $\mu_{e}=$ $I / \rho$ where

$$
I=\int_{0}^{1} \frac{1-s}{f(s)-s} d s
$$

and hence $\mathcal{S}$ is transient if $I<\infty$ and it is null-recurrent if $I=\infty$. This is equivalent to Theorem 3.2 in [2]. Since $f(s)-$ $s=(1-m)(1-s)(1+o(1))$ as $s \rightarrow 1-$, it is obvious that $I$ is finite if $m<1$; that is, $\mathcal{S}$ is transient. The typical case for $m=1$ is that the variance $2 \gamma:=f^{\prime \prime}(1)$ is finite. In this case $f(s)-s \sim(1-s)^{2} \gamma$, and we see that $I=\infty$; that is, $\mathcal{S}$ is 
null-recurrent. It is well known that there are examples where $\gamma=\infty$ and $I<\infty$.

More detail about the asymptotic behaviour of $P(t)$ can be extracted from known results about $\mathscr{F}(t)$. A clue as to what can be expected is exposed by letting $s \rightarrow 1$ in (26). This yields

$$
\sum_{i \geq 0} p_{i j}(t)=E_{1}\left(Z_{t}\right)=e^{-\alpha t}
$$

where $\alpha=(1-m) \rho$ denotes the Malthusian parameter of the MBP. This result shows that the uniform measure on $\mathcal{S}$ is $\alpha$-invariant for $P(t)$. See Section 5.2 in [6] for this notion. If $m<1$ then $\alpha>0$, and it is known that

$$
1-F(t)=e^{-\alpha t} L\left(e^{\alpha t}\right)
$$

where $L(x)$ is slowly varying at infinity and $0<L(\infty)<\infty$ if $\sum_{j \geq 1} p_{j} j \log j<\infty$, and $L(\infty)=0$ otherwise. In addition

$$
\lim _{t \rightarrow \infty} \frac{F(s, t)-F(t)}{1-F(t)}=\mathscr{M}(s)=\sum_{j \geq 1} m_{j} s^{j},
$$

where $\mathscr{M}(s)$ is a nondefective probability generating function and $m_{j}>0$ for all $j \geq 1$. See p. 121 in [11] for these facts. These $m_{j}$ 's comprise $\alpha$-invariant measure for $\mathscr{F}(t)$ (restricted to $\mathbb{N}$ ), which may be expressed in terms of generating functions as

$$
1-\mathscr{M}(F(s, t))=e^{-\alpha t}(1-\mathscr{M}(s)) .
$$

The generating function of the tail masses $x_{i}=\sum_{k>i} m_{k}$ is $(1-\mathscr{M}(s)) /(1-s)$.

Theorem 5. Let $m<1$. Then $\left\{x_{i}\right\}$ is $\alpha$-invariant function for $P(t)$ and, as $t \rightarrow \infty$,

$$
\lim _{t \rightarrow \infty} \frac{p_{i j}(t)}{1-F(t)}=x_{i}, \quad(i, j \geq 0) .
$$

Proof. Using (26) we compute

$$
\begin{aligned}
\sum_{j \geq 0} G_{j}(s, t) x_{j} & =G_{0}(s, t) \sum_{j \geq 0}(F(s, t))^{j} x_{j} \\
& =G_{0}(s, t) \frac{1-\mathscr{M}(F(s, t))}{1-F(s, t)} \\
& =e^{-\alpha t} \frac{1-\mathscr{M}(s)}{1-s}
\end{aligned}
$$

by virtue of (26) with $j=0$ and (64), and the $\alpha$-invariance assertion follows. The asymptotic relation follows because (26) and (63) imply that

$$
\lim _{t \rightarrow \infty} \frac{G_{j}(s, t)}{1-F(t)}=\frac{1-\mathscr{M}(s)}{1-s},
$$

and the extended continuity theorem then yields the assertion.

Turning to the case $m=1$, we have the following result similar to Theorem 5 .
Theorem 6. If $m=1$ then

$$
\lim _{t \rightarrow \infty} \frac{p_{i j}(t)}{1-F(t)}=1, \quad(i, j \geq 0) .
$$

Proof. The assertion follows once we show that $G_{j}(s, t) /(1-$ $F(t)) \rightarrow(1-s)^{-1}$, and this will follow from

$$
\lim _{t \rightarrow \infty} \frac{F(s, t)-F(t)}{1-F(t)}=0 .
$$

This is quite well known, and it can be shown easily as follows. Let $s<1$ and choose $u>0$ such that $s=F(u)$. This is possible since $F(0)=0$ and $F(t)$ is a continuous distribution function. Then $F(s, t)=F(u+t)=F(F(t), u)$, and hence the limit (69) is

$$
\lim _{t \rightarrow \infty} \frac{F(F(t), u)-F(t)}{1-F(t)}=\lim _{z \rightarrow 1} \frac{F(z, u)-z}{1-z}=0,
$$

since $F^{\prime}(1-, u)=E_{1}\left(Z_{u}\right) \equiv 1$.

We can obtain a second-order correction to this result by using the following further facts about the MBP. Recall that $a(s)=(f(s)-s) \rho$, and observe that since $(1-f(s)) /(1-s)$ is a probability generating function if $m=1$; then $1 /(f(s)-s)$ has a Maclaurin expansion with nonnegative coefficients. Hence the function $V(s)=\int_{0}^{s} d u / a(u)=\sum_{j \geq 1} v_{j} s^{j}$ is finite if $|s|<1$, $V(1-)=\infty$, and $v_{j} \geq 0$. The integrated backward equation (15) can be expressed as

$$
V(F(s, t))-V(s)=t .
$$

Setting $s=0$ shows that $F(t)$ is the inverse function of $V$ and that $F(s, t)=F(t+V(s))$. It follows too from (71) that $\sum_{i \geq 1} v_{i} f_{i j}(t)=v_{j}$; that is, $\left\{v_{j}: j \geq 1\right\}$ is an invariant measure for $\mathscr{F}(t)$ restricted to $\mathbb{N}$. It is known to be unique up to multiplication by constants. The following proposition expresses in terms of $F(t)$ the precise rate at which $F(s, t)-$ $F(t)$ tends to zero.

Proposition 7. The limit

$$
\lim _{t \rightarrow \infty} \frac{F(s, t)-F(t)}{F(t+1)-F(t)}=V(s) .
$$

Proof. A Taylor expansion of $f(F(s, t))$ about $F(t)$ yields

$$
\begin{aligned}
\frac{a(F(t))-a(F(s, t))}{F(t+1)-F(t)}=1-f^{\prime}(F(t))+o(1), & \\
& (t \longrightarrow \infty) .
\end{aligned}
$$

Since $a(s)$ is a decreasing function, it follows from (15) and then (71) that

$$
\int_{F(t)}^{F(s, t)} \frac{d u}{a(u)}=V(s),
$$

and hence that

$$
\frac{F(s, t)-F(t)}{a(F(t))}<V(s)<\frac{F(s, t)-F(t)}{a(F(s, t))} .
$$


If at least one extreme side of this inequality is bounded away from $V(s)$ as $t \rightarrow \infty$ through some sequence, then (73) will be violated. It follows that the limit as $t \rightarrow \infty$ of each bound exists and equals $V(s)$. Setting $s=F(1)$, then since $F(F(1), t)=F(t+1)$, we conclude that

$$
\lim _{t \rightarrow \infty} \frac{F(t+1)-F(t)}{a(F(t))}=V(F(1))=1,
$$

and the assertion follows.

Let $\sigma_{0}=0$ and $\sigma_{i}=\sum_{k=1}^{i} v_{k}$ for $i \geq 1$. Then $\sum_{i \geq 1} \sigma_{i} s^{i}=$ $V(s) /(1-s)$.

Theorem 8. If $m=1$ and $2 \gamma=f^{\prime \prime}(1-) \leq \infty$, then

$$
\lim _{t \rightarrow \infty} \frac{1-F(t)-p_{i j}(t)}{F(t+1)-F(t)}=\sigma_{i}+\frac{j}{\gamma \rho} .
$$

Proof. The generating function of the term in the numerator is

$$
\begin{aligned}
& \frac{1-F(t)}{1-s}-G_{j}(s, t) \\
& \quad=\frac{F(s, t)-F(t)+(1-F(s, t))\left(1-(F(s, t))^{j}\right)}{1-s} .
\end{aligned}
$$

Dividing by $F(t+1)-F(t)$ and letting $t \rightarrow \infty$, it follows from Proposition 7 that the first term in the right-hand side numerator contributes the limit $V(s) /(1-s)$. The second term in that numerator is asymptotically equal to $(1-F(s, t))^{2} j$. Choosing $u>0$ such that $s=F(u)$, we find that

$$
\frac{(1-F(s, t))^{2}}{F(t+1)-F(t)}=\frac{(1-F(F(t), u))^{2}}{F(F(t), 1)-F(t)},
$$

so a double application of L'Hopitâl's rule shows that the limit of the right-hand side, as $t \rightarrow \infty$, equals $2\left(F^{\prime}(1, u)\right)^{2} / F^{\prime \prime}(1,1)=(\gamma \rho)^{-1}$ since $F^{\prime}(1, u)=E_{1}\left(Z_{u}\right) \equiv 1$, and $F^{\prime \prime}(1,1)=\operatorname{Var}_{1}\left(Z_{1}\right)=a^{\prime \prime}(1)=2 \gamma \rho$. See p. 109 in [10] for the moment formulae. The assertion follows from these limit identities.

If $\gamma<\infty$ then it can be shown using (33) that $t^{2}(F(t+1)-$ $F(t)) \rightarrow\left(\rho \gamma^{2}\right)^{-1}$, thus giving a precise estimate of the second order correction supplied by Theorem 8 .

\section{Limit Theorems}

In this section we assume that $m \leq 1$ and, if $m=1$, then we shall understand (63) to hold with $\mathscr{M}(s) \equiv 0$. Our first result shows that $\xi_{t}:=(1-F(t)) X_{t}$ converges in law. We denote this mode of convergence by $\stackrel{d}{\rightarrow}$.

Theorem 9. (a) If $m=1$ then, for all $i \geq 0, \xi_{t} \stackrel{d}{\rightarrow} \varepsilon$, where $\varepsilon$ has the standard exponential law.

(b) If $m<1$ and $X_{0}=i$, then $\xi_{t} \stackrel{d}{\rightarrow} W$, where the law of $W$ is a finite mixture of Erlang laws, having the density function (85) below.
Proof. It follows from (26) that

$$
\sum_{i \geq 0} s^{i} E_{i}\left(e^{-\theta X_{t}}\right)=\frac{1-F(s, t)}{(1-s)\left(1-e^{-\theta} F(s, t)\right)} .
$$

Replacing $\exp (-\theta)$ with $\exp [-(1-F(t)) \theta]=1-(1-F(t)) \theta(1+$ $o(t))$, it follows from (63) that

$$
\lim _{t \rightarrow \infty} \sum_{i \geq 0} s^{i} E_{i}\left(e^{-\theta \xi_{t}}\right)=\frac{1-\mathscr{M}(s)}{(1-s)(1-\mathscr{M}(s)+\theta)} .
$$

Assertion (a) follows from the extended continuity theorem for probability generating functions and $(1+\theta)^{-1}$ is the Laplace-Stieltjes transform of the standard exponential law.

If $m<1$ then the right-hand side of (81) can be expanded in the form

$$
\frac{1-\mathscr{M}(s)}{1-s}(1+\theta)^{-1} \sum_{j \geq 0}\left(\frac{\mathscr{M}(s)}{1+\theta}\right)^{j} .
$$

But

$$
\frac{1-\mathscr{M}(s)}{1-s}(\mathscr{M}(s))^{j}=\sum_{i \geq 0} s^{i} r_{i j},
$$

where $r_{i j}$ is the one-step transition probability of the Markov chain which is the (modified) Siegmund dual of the simple branching process whose offspring probability generating function is $\mathscr{M}(s)$. This offspring law is supercritical, and, indeed, since $\mathscr{M}(0)=0$, it follows that $r_{i j}=0$ if $j>i \geq 0$. See [3] for properties of this dual. We conclude from (81) that

$$
\lim _{t \rightarrow \infty} E_{i}\left(e^{-\theta \xi_{t}}\right)=(1+\theta)^{-1} \sum_{j=0}^{i} r_{i j}(1+\theta)^{-j}
$$

and hence that Assertion (b) holds, where $W$ has the LaplaceStieltjes transform on the right-hand side of (84), and its density function is

$$
g_{i}(x)=e^{-x} \sum_{j=0}^{i} r_{i j} \frac{x^{j}}{j !}, \quad(i, x \geq 0) .
$$

This completes the proof.

We show next that the the mode of convergence in Theorem 9 (b) can be strengthened to almost sure convergence. Our proof exploits the upward skip-free nature of DMBP sample paths. It follows quite closely the progression through Theorems 2.2 to 2.4 in [19], which in turn is an application of basic methodology developed in [20].

Theorem 10. If $m<1$ and $X_{0}=i$, then $\xi_{t} \stackrel{\text { a.s. }}{\longrightarrow} W$.

Proof. Let $T_{i j}$ be the hitting time of state $j$ by the DMBP. The skip-free property of its sample paths implies that if $j>X_{0}=$ $i$ then $T_{i j}=\sum_{k=i+1}^{j} \tau_{k}$, where $\tau_{k}$ is the first-passage time from $k-1$ to $k$, and $\tau_{k}$ 's are independent. Writing $T_{j}=T_{0 j}$, the quantities

$$
x(j ; \theta):=\left[E_{0}\left(e^{-\theta T_{j}}\right)\right]^{-1}, \quad(j \geq 0)
$$


are computed by solving the linear system

$$
\sum_{j \geq 0} q_{i j} x(j ; \theta)=\theta x(i ; \theta), \quad(i \geq 0) .
$$

It is shown in [20] that this system has the stated solution and it is unique up to constant factors. Indeed, $x(0 ; \theta) \equiv 1$, and starting with $i=0,1, \ldots$, the system can be solved recursively for $x(1 ; \theta), x(2 ; \theta), \ldots$.

Formally define the generating function $X(s)=$ $\sum_{i \geq 0} x(i ; \theta) s^{i}$. Recalling that $a(s)=(f(s)-s) \rho$, it follows from (4) and (33) that

$$
\sum_{i \geq 0} s^{i} q_{i j}=\left(j s^{j-1}-(j+1) s^{j}\right) a(s),
$$

which together with (87) yields the differential equation

$$
X^{\prime}(s)-\left(\frac{\theta}{a(s)}+\frac{1}{1-s}\right) X(s)=0 .
$$

Integration yields the solution

$$
X(s)=(1-s)^{-1} e^{\theta V(s)}
$$

where, we recall, $V(s)=\int_{0}^{s} d u / a(u)$. The right-hand side has a Maclaurin expansion whose coefficients are positive and which satisfies system (87). The function $\mathscr{M}(s)$ defined at (63) is related to $V(s)$ by

$$
1-\mathscr{M}(s)=e^{-\alpha V(s)}=\frac{(1-s)}{L\left((1-s)^{-1}\right)},
$$

where $L(x)$ is slowly varying at infinity; see p. 122 in [11] for the right-hand side representation. It follows that

$$
X(s)=(1-s)^{-1-\theta / \alpha}\left[L\left((1-s)^{-1}\right)\right]^{\theta / \alpha} .
$$

Since $T_{j}$ is almost surely nondecreasing in $j$, then $x(j)$ is nondecreasing and hence a Tauberian theorem for power series yields the asymptotic equivalence

$$
x(j ; \theta) \sim \frac{[j L(j)]^{\theta / \alpha}}{\Gamma(1+\theta / \alpha)}
$$

in other words, as $j \rightarrow \infty$ we have the asymptotic equivalence

$$
E_{0}\left(e^{-\theta T_{j}}\right) \sim \Gamma\left(1+\frac{\theta}{\alpha}\right)[j L(j)]^{-\theta / \alpha} .
$$

For $x \geq 1$, define $\gamma(x)=V(1-1 / x)$ and observe that (91) takes the form

$$
R(x):=\exp [\gamma(x)]=(x L(x))^{1 / \alpha} .
$$

It follows that (94) can be expressed as

$$
\lim _{j \rightarrow \infty} E_{0}\left(e^{-\theta\left(T_{j}-\gamma(j)\right)}\right)=\Gamma\left(1+\frac{\theta}{\alpha}\right) .
$$

The right-hand side is the (bilateral) Laplace-Stieltjes transform of a random variable $S$ which has the Gumbel type distribution function $\exp \left(-e^{-\alpha x}\right)$. We have thus shown that $T_{j}-\gamma(j) \stackrel{d}{\rightarrow} S$. But the left-hand side is a sum of independent random variables, so it follows from the equivalence theorem for convergent random series of independent summands that $T_{j}-\gamma(j) \stackrel{\text { a.s. }}{\longrightarrow} S$. surely,

Recalling that $T_{i j}=\sum_{k=i+1}^{j} \tau_{k}$, it follows that, $P_{i}$-almost

$$
T_{i j}-\gamma(j) \longrightarrow S_{i},
$$

where $S=S_{i}+T_{i}$ and $S_{i}$ and $T_{i}$ are independent. Hence the Laplace-Stieltjes transform of the limit is

$$
E\left(e^{-\theta S_{i}}\right)=x(i ; \theta) \Gamma\left(1+\frac{\theta}{\alpha}\right), \quad(\theta \geq 0) .
$$

Next, let $\mathscr{F}_{t}=\sigma\left\{X_{v}: v \leq t\right\}$. Then

$$
M_{t}=E\left(e^{-\theta S_{i}} \mid \mathscr{F}_{t}\right)
$$

is a positive-valued Lévy martingale, and $M_{t} \stackrel{\text { a.s. }}{\longrightarrow} e^{-\theta S_{i}}$. On the other hand,

$$
\begin{aligned}
M_{t} & =\lim _{j \rightarrow \infty} E_{i}\left[e^{-\theta\left(T_{i j}-\gamma(j)\right)} \mid \mathscr{F}_{t}\right] \\
& =e^{-\theta t} \lim _{j \rightarrow \infty} E_{X_{t}}\left[e^{-\theta\left(T_{X_{t}, j}-\gamma(j)\right)} \mid \mathscr{F}_{t}\right] \\
& =e^{-\theta t} E\left[e^{-\theta S_{X_{t}}}\right]=e^{-\theta t} x\left(X_{t} ; \theta\right) \Gamma\left(1+\frac{\theta}{\alpha}\right),
\end{aligned}
$$

where we have used the strong Markov property for the second equality, (97) and the dominated convergence theorem for the penultimate equality and then (98). Letting $\theta=1$ in this result, (93) and (98) show that if $t \rightarrow \infty$, then almost surely

$$
M_{t} \sim e^{-t}\left[\frac{e^{\gamma\left(X_{t}\right)}}{\Gamma(1+1 / \alpha)}\right] \Gamma\left(1+\frac{1}{a}\right) \longrightarrow e^{-S_{i}}
$$

that is, $t-\gamma\left(X_{t}\right) \rightarrow S_{i}, P_{i}$-almost surely. This conclusion can be expressed as the asymptotic equivalence $R\left(X_{t}\right) \sim e^{t-S_{i}}$. Since $R(x)$ is regularly varying with index $\alpha^{-1}$, its inverse $\varphi(y)$ is regularly varying with index $\alpha$, and hence

$$
X_{t} \sim \varphi\left(e^{t-S_{i}}\right) \sim e^{-\alpha S_{i}} \varphi\left(e^{t}\right) .
$$

The assertion follows after some algebra to check that the definitions of $V, R$, and $\varphi$ imply that $\varphi\left(e^{t}\right)=1 /(1-F(t))$.

If $m<1$ then the condition $\sum_{j \geq 1} p_{j} j \log j<\infty$ is equivalent to

$$
\phi:=\int_{0}^{1}\left[\frac{1}{1-s}-\frac{1-m}{f(s)-s}\right] d s<\infty .
$$

Assuming this, it follows that

$$
V(s)=\alpha^{-1}[-\log (1-s)-\phi+o(1)], \quad(s \longrightarrow 1) .
$$


Consequently, the norming in Theorem 9 has the asymptotic form $1-F(t) \sim e^{-\alpha t-\phi}$. In addition, $\gamma(x)=\alpha^{-1}(\log x-\phi)+$ $o(1)$ as $x \rightarrow \infty$, and hence the norming function emerging in the final stages of the previous proof satisfies $\varphi\left(e^{t}\right) \sim e^{\alpha t+\phi}$. These equivalences show that the limit laws in Theorems 9 and 10 agree, and we have the identification $W=e^{-\alpha S_{i}} P_{i^{-}}$ almost surely.

We will state and prove two results concerning the rate at which $\xi_{t}$ converges to $W$. First we recollect some known results concerning $\mathscr{M}(s)$ defined by (63). If we let $s=0$ in (64), then differentiating with respect to $t$ and recalling (14) yield

$$
a(s) \mathscr{M}^{\prime}(s)=\alpha(1-\mathscr{M}(s)) .
$$

Integration leads to the identity

$$
\frac{1-\mathscr{M}(s)}{1-s}=\exp \left(\int_{0}^{s}\left(\frac{1}{1-u}-\frac{1-m}{f(u)-u}\right) d u\right) .
$$

It follows that the mean of the limiting conditional law for the MBP is $\mu=e^{\phi}$. We will assume that $f^{\prime \prime}(1)$ is finite, in which case differentiating (105) leading to

$$
\kappa:=\mathscr{M}^{\prime \prime}(1)=\frac{\mu f^{\prime \prime}(1)}{1-m}
$$

is finite. Hence we have the expansion

$$
1-\mathscr{M}(s)=(1-s) \mu-\frac{(1-s)^{2} \kappa}{2}+o\left((1-s)^{2}\right) .
$$

Finally, we let $\sigma^{2}=1+\kappa / \mu$.

Theorem 11. If $m<1$ and $f^{\prime \prime}(1)<\infty$, then

$$
\frac{\xi_{t}-W}{\sqrt{1-F(t)}} \stackrel{d}{\longrightarrow} \sigma \Xi
$$

where the law of $\Xi$ is the finite mixture of generalized Laplace laws whose characteristic function is

$$
\omega(\zeta):=E\left(e^{i \zeta \Xi}\right)=\sum_{j=0}^{i} r_{i j}\left(1+\frac{1}{2} \zeta^{2}\right)^{-j-1} .
$$

Proof. First we derive the Laplace-Stieltjes transform of $W-$ $\xi_{t}$. The Markov property implies that

$$
\begin{aligned}
& E_{i}\left(e^{-\theta \xi_{t+u}} \mid \mathscr{F}_{t}\right)=E_{X_{t}}\left(\exp \left[-\theta \frac{1-F(t+u)}{1-F(t)} \xi_{u}\right]\right) \\
& \longrightarrow E_{X_{t}}\left(\exp \left[-\theta e^{-\alpha t} W\right]\right), \\
&(u \longrightarrow \infty) .
\end{aligned}
$$

Let $\tau=\exp [\theta(1-F(t))]$, and suppose that $\theta$ is restricted to imaginary values. Multiplying through by $e^{\theta \xi_{t}}$ and using the dominated convergence theorem we obtain the identity

$$
\begin{aligned}
\psi_{i}(\theta, t) & :=E_{i}\left(e^{-\theta\left(W-\xi_{t}\right)}\right) \\
& =E_{i}\left[\tau^{X_{t}} E_{X_{t}}\left(\exp \left[-\theta e^{-\alpha t} W\right]\right)\right] \\
& =\sum_{j \geq 0} p_{i j}(t) \tau^{j} E_{j}\left(\exp \left[-\theta e^{-\alpha t} W\right]\right) .
\end{aligned}
$$

It follows from (26) and (84) that

$$
\begin{aligned}
& \sum_{i \geq 0} s^{i} \psi_{i}(\theta, t) \\
& =G_{0}(s, t) \sum_{j \geq 0}(\tau F(s, t))^{j} E_{j}\left(\exp \left[-\theta e^{-\alpha t} W\right]\right) \\
& =G_{0}(s, t) \frac{1-\mathscr{M}(\tau F(s, t))}{[1-\tau F(s, t)]\left[\theta e^{-\alpha t}+1-\mathscr{M}(\tau F(s, t))\right]} .
\end{aligned}
$$

Next, replace $\theta$ with $\theta_{t}=\theta / \sqrt{1-F(t)}$. Since $\tau F(s, t) \rightarrow 1$ as $t \rightarrow \infty$, the factor

$$
\frac{1-\mathscr{M}(\tau F(s, t))}{1-\tau F(s, t)} \longrightarrow \mu .
$$

Using (64) with $s=0$ and then (108), we have

$$
\begin{aligned}
\theta_{t} e^{-\alpha t} & =\frac{1-\mathscr{M}(F(t))}{\sqrt{1-F(t)}} \theta \\
& =\mu \theta \sqrt{1-F(t)}+O\left((1-F(t))^{3 / 2}\right) .
\end{aligned}
$$

Expanding $\tau$ gives

$$
\begin{aligned}
1-\tau F(s, t)= & 1-F(s, t)-\theta \sqrt{1-F(t)} \\
& -\frac{1}{2} \theta^{2}(1-F(t))(1+o(1)) .
\end{aligned}
$$

So it follows from (108) that

$$
\begin{aligned}
1-\mathscr{M}(\tau F(s, t))= & \mu(1-F(s, t))-\mu \theta \sqrt{1-F(t)} \\
& -\frac{1}{2} \mu \sigma^{2} \theta^{2}(1-F(t)) \\
& +o(1-F(t)) .
\end{aligned}
$$

Adding this to (115) cancels terms in $\sqrt{1-F(t)}$. It follows from (113), (26) with $j=0$, and (114) that

$$
\begin{aligned}
& \sum_{i \geq 0} s^{i} \psi_{i}(\theta, t) \\
& \quad=\frac{(1-F(s, t))(1+o(1))}{(1-s)\left[1-F(s, t)-(1 / 2) \sigma^{2} \theta^{2}(1-F(t))(1+o(1))\right]} \\
& \quad \longrightarrow \frac{1-\mathscr{M}(s)}{(1-s)\left(1-\mathscr{M}(s)-(1 / 2) \sigma^{2} \theta^{2}\right)} .
\end{aligned}
$$

Writing $\theta=-i \zeta$, where $\zeta$ is real-valued, it follows as in the proof of Theorem 9 that the characteristic function $\omega(\zeta, t)$ of $\left(\xi_{t}-W\right) / \sqrt{1-F(t)}$ converges to $\omega(\sigma \zeta)$, as asserted. This completes the proof.

The term $\left(1+(1 / 2) \zeta^{2}\right)^{-j-1}$ in (110) is the characteristic function of the sum of $j+1$ independent copies of $2^{-1 / 2} \Lambda$, where $\Lambda$ has a standard Laplace law. If $\Lambda_{1}, \Lambda_{2}, \ldots$ are independent copies of $\Lambda$, then $\Lambda_{1}+\cdots+\Lambda_{j+1}=\gamma_{j+1}^{\prime}-\gamma_{j+1}^{\prime \prime}$, where $\gamma_{j+1}$ 's are independent and have the Erlang density 
$x^{j} e^{-x} / j$ ! for $x \geq 0$. It follows that the density of $\gamma_{j+1}^{\prime}-\gamma_{j+1}^{\prime \prime}$ is

$$
\begin{aligned}
f_{j}(x) & =\left(\frac{e^{-x}}{(j !)^{2}}\right) \int_{0}^{\infty}(x+y)^{j} y^{j} e^{-2 y} d y \\
& =(k ! \sqrt{\pi})^{-1}\left(\frac{1}{2} x\right)^{j+1 / 2} B_{j+1 / 2}(x),
\end{aligned}
$$

where

$$
B_{\nu}(x)=\frac{2}{\pi} \int_{0}^{(1 / 2) \pi} \cos (x \tan \theta-\nu \theta) d \theta
$$

is Bateman's function, and the evaluation is Entry 3.38.8 in Gradshteyn and Ryzhik [21]. Hence the limit law has the density function $(\sqrt{2} / \sigma) \sum_{j=0}^{i} r_{i j} f_{j}(\sqrt{2} x / \sigma)$, which is a Laplace law density function if $i=0$.

Let $\mathcal{N}$ have a standard normal law and be independent of the above $\gamma_{j+1}$ 's. Then the characteristic function of $\mathcal{N} \sqrt{\gamma_{j+1}}$ is $\left(1+(1 / 2) \zeta^{2}\right)^{-j-1}$, and hence $\Xi \stackrel{d}{=} \mathcal{N} \sqrt{W}$, where $W$ is as defined in Theorem 9(b), and it is independent of $\mathcal{N}$. It follows that it ought to be possible to simplify the mixed limit law in Theorem 11 by replacing the norming function with $\sqrt{\xi_{t}}$. The following result achieves this. To see why, let $c_{t}=1 /(1-F(t))$ and observe that Theorem 11 can be expressed as $\left(X_{t}-c_{t} W\right) / \sqrt{c_{t}} \stackrel{d}{\rightarrow} \sigma \mathscr{N} \sqrt{W}$. Replacing $c_{t}$ in the norming by $X_{t}$ will eliminate this $\sqrt{W}$ factor from the weak limit.

Recall from the end of the last proof that, under the second-order moment assumption assumed in the following result, $c_{t} \sim e^{\alpha t+\phi}$.

Theorem 12. If $m<1$ and $f^{\prime \prime}(1)<\infty$, then

$$
\frac{X_{t}-c_{t} W}{\sqrt{X_{t}}} \stackrel{d}{\longrightarrow} \sigma \mathcal{N} \text {. }
$$

Proof. We begin by refining this $o(1)$ remainder term in the expression for $\gamma(i)$ which we deduced from (104). The second moment condition allows us to write

$$
\frac{1-m}{f(s)-s}=\frac{1-m}{(1-m)(1-s)(1+O(1-s))},
$$

so

$$
\frac{1}{1-s}-\frac{1-m}{f(s)-s}=\frac{O(1)}{1+O(1-s)} .
$$

Consequently

$$
\int_{1-1 / i}^{1}\left(\frac{1}{1-s}-\frac{1-m}{f(s)-s}\right) d s=O\left(\frac{1}{i}\right),
$$

and hence

$$
\begin{aligned}
\gamma(i) & =\int_{0}^{1-1 / i} \frac{d u}{a(u)} \\
& =\alpha^{-1}\left[\log i-\int_{0}^{1-1 / i}\left(\frac{1}{1-s}-\frac{1-m}{f(s)-s}\right) d s\right] \\
& =\alpha^{-1}(\log i-\phi)+O\left(\frac{1}{i}\right) .
\end{aligned}
$$

For $j \geq 1$ let $v(j)=\gamma(j)-\gamma(j-1)$ and observe that $S=\sum_{r \geq 1}\left(\tau_{r}-v(r)\right)$, and hence

$$
S_{i}=S-T_{i}=\sum_{r>i}\left(\tau_{r}-v(r)\right)-\gamma(i)
$$

It follows that if $j>i$, then $T_{i j}-\gamma(j)=\sum_{r=i+1}^{j}\left(\tau_{r}-v(r)\right)-\gamma(i)$; that is,

$$
T_{i j}-\gamma(j)-S_{i}=-\left(S_{j}+\gamma(j)\right)=-\sum_{r>j}\left(\tau_{r}-v(r)\right) .
$$

We prove asymptotic normality of the right-hand side as follows. We find from (98) that

$$
\begin{aligned}
\sum_{i \geq 0} s^{i} E\left(e^{-\theta S_{i}}\right) & =\frac{X(s)}{1-s} \Gamma\left(1+\frac{\theta}{\alpha}\right) \\
& =(1-s)^{-1} E\left[e^{-\theta\left(\alpha^{-1} U-V(s)\right)}\right],
\end{aligned}
$$

where $U$ has a standard Gumbel law. Inverting the LaplaceStieltjes transforms gives the identity

$$
\begin{aligned}
\sum_{i \geq 0} s^{i} P\left(S_{i} \leq z\right) & =(1-s)^{-1} P(U \leq \alpha(z+V(s))) \\
& =(1-s)^{-1} \exp \left[-e^{-\alpha z-V(s)}\right] \\
& =(1-s)^{-1} \exp \left[-e^{-\alpha z}(1-\mathscr{M}(s))\right],
\end{aligned}
$$

since $\alpha V(s)=\int_{0}^{s}((1-m) /(f(u)-u)) d u$, and we have used (91). The exponential factor in (129) is the probability generating function of a random variable, $C_{z}$ say, which has a compound Poisson law. We thus obtain the representation

$$
P\left(S_{j} \leq z\right)=P\left(C_{z} \leq j\right)
$$

Let $z=z(j):=y / \sqrt{j}-\gamma(j)$, and denote the random infinite series in (127) by $R_{j}$. The representation then takes the form

$$
\begin{aligned}
& P\left(\sqrt{j}\left(S_{j}+\gamma(j)\right) \leq y\right)=P\left(\sqrt{j} R_{j}\right) \\
& \quad=P\left(C_{z(j)} \leq j\right)=P\left(\frac{\left(C_{z(j)}-j\right)}{\sqrt{j}} \leq 0\right),
\end{aligned}
$$

and we evaluate the limit of the right-hand side as follows.

Noting that $E\left(C_{z}\right)=e^{-\alpha z} \mathscr{M}^{\prime}(1)=\mu e^{-\alpha z}$, and recalling that $\mu=e^{\phi}$, we find from (125) that

$$
\begin{aligned}
b(j) & :=E\left(C_{z(j)}\right)=j e^{\phi-\phi+O(1 / j)-\alpha y / \sqrt{j}} \\
& =j-\alpha y \sqrt{j}+O(1) .
\end{aligned}
$$


It follows from (108) that

$$
\begin{aligned}
& -\log E\left[e^{-\theta\left(C_{z(j)}-j\right) / \sqrt{j}}\right] \\
& =-\theta \sqrt{j}+e^{-\alpha z(j)}\left(1-\mathscr{M}\left(\exp \left(-\frac{\theta}{\sqrt{j}}\right)\right)\right) \\
& =-\theta \sqrt{j}+b(j)\left(\frac{\theta}{\sqrt{j}}-\frac{\theta^{2}}{2 j}-\frac{\kappa \theta^{2}}{2 \mu j}+o\left(j^{-1}\right)\right) \\
& =-\theta \sqrt{j}+\theta \sqrt{j}-\alpha y \theta-\frac{1}{2} \sigma^{2} \theta^{2}+o(1) \\
& \longrightarrow-\alpha y \theta-\frac{1}{2} \sigma^{2} \theta^{2} .
\end{aligned}
$$

This implies that $\left(C_{z(j)}-j\right) / \sqrt{j} \stackrel{d}{\rightarrow} \sigma \mathcal{N}-\alpha y$, and hence that

$$
P\left(\sqrt{j} R_{j} \leq y\right) \rightarrow P(\sigma \mathcal{N}-\alpha y \leq 0)
$$

Thus we have shown that

$$
\sqrt{j}\left(T_{i j}-\gamma(j)-S_{i}\right) \stackrel{d}{\longrightarrow} \frac{\sigma \mathcal{N}}{\alpha} .
$$

Theorem 4.1 in [20] asserts that this is equivalent to the following assertion: if $X_{0}=i$, then

$$
\sqrt{X_{t}}\left(t-\gamma\left(X_{t}\right)-S_{i}\right) \stackrel{d}{\longrightarrow} \frac{\sigma \mathcal{N}}{\alpha} .
$$

Using (125), we write this more explicitly as

$$
A_{t}:=\sqrt{X_{t}}\left(\alpha t+\phi-\log X_{t}-\alpha S_{i}\right) \stackrel{d}{\longrightarrow} \sigma \mathcal{N} .
$$

We have seen, under the second-order moment condition, that Theorem 10 can be expressed as $e^{-\alpha t-\phi} X_{t} \rightarrow e^{-a S_{i}}\left(P_{i^{-}}\right.$ almost surely). So

$$
\begin{aligned}
D_{t} & :=X_{t}-e^{\alpha t+\phi-\alpha S_{i}}=X_{t}\left(1-e^{A_{t} / \sqrt{X_{t}}}\right) \\
& =-A_{t} \sqrt{X_{t}}+O_{p}\left(A_{t}^{2}\right) .
\end{aligned}
$$

Hence

$$
\frac{D_{t}}{\sqrt{X_{t}}}=-A_{t}+O_{p}\left(\frac{A_{t}^{2}}{\sqrt{X_{t}}}\right) \stackrel{d}{\longrightarrow} \sigma \mathcal{N},
$$

as asserted.

\section{Conflicts of Interest}

The author declares that there are no conflicts of interest regarding the publication of this paper.

\section{References}

[1] D. Siegmund, "The equivalence of absorbing and reflecting barrier problems for stochastically monotone Markov processes," The Annals of Probability, vol. 4, no. 6, pp. 914-924, 1976.
[2] Y. Li, A. G. Pakes, J. Li, and A. Gu, "The limit behavior of dual Markov branching processes," Journal of Applied Probability, vol. 45, no. 1, pp. 176-189, 2008.

[3] A. G. Pakes, "The limit behaviour of a Markov chain related to the simple branching process allowing immigration," Journal of Mathematical and Physical Sciences, vol. 15, pp. 159-171, 1980.

[4] A. G. Pakes, “The Galton-Watson process with killing," Mathematical Biosciences, vol. 69, no. 2, pp. 171-188, 1984.

[5] A. Chen, P. Pollett, H. Zhang, and B. Cairns, "Uniqueness criteria for continuous-time Markov chains with general transition structures," Advances in Applied Probability, vol. 37, no. 4, pp. 1056-1074, 2005.

[6] W. J. Anderson, Continuous-Time Markov Chains, Springer, New York, NY, USA, 1991.

[7] T. E. Harris, The theory of branching processes, vol. 119 of Die Grundlehren der Mathematischen Wissenschaften, Bd. 119, Springer, Berlin, Germany, 1 edition, 1963.

[8] Z. T. Hou and Q. F. Guo, Homogeneous Denumerable Markov Processes, Springer, Berlin, Germany, 1988.

[9] A. G. Pakes, "Explosive Markov branching processes: entrance laws and limiting behaviour," Advances in Applied Probability, vol. 25, no. 4, pp. 737-756, 1993.

[10] K. B. Athreya and P. E. Ney, Branching processes, Springer, Berlin, Germany, 1972.

[11] S. Asmussen and H. Hering, Branching Processes, Birkhäuser, Boston, Mass, USA, 1983.

[12] K. B. Athreya and S. Karlin, "Limit theorems for the split times of branching processes," Journal of Mathematics and Mechanics, vol. 17, pp. 257-277, 1967.

[13] A. Chen, "Applications of Feller-Reuter-Riley transition functions," Journal of Mathematical Analysis and Applications, vol. 260, no. 2, pp. 439-456, 2001.

[14] A. G. Pakes, "Extinction and explosion of nonlinear Markov branching processes," Journal of the Australian Mathematical Society, vol. 82, no. 3, pp. 403-428, 2007.

[15] S. Karlin and S. Tavaré, "Linear birth and death processes with killing," Journal of Applied Probability, vol. 19, no. 3, pp. 477-487, 1982.

[16] Y. Li, "Dual and Feller-Reuter-Riley transition functions," Journal of Mathematical Analysis and Applications, vol. 313, no. 2, pp. 461-474, 2006.

[17] L. Breiman, Probability, Addison-Wesley Publishing Company, Reading, MA, USA, 1968.

[18] H. Zhang, A. Chen, X. Lin, and Z. Hou, "Strong ergodicity of monotone transition functions," Statistics and Probability Letters, vol. 55, no. 1, pp. 63-69, 2001.

[19] A. G. Pakes, "The supercritical birth, death and catastrophe process: limit theorems on the set of non-extinction," Journal of Mathematical Biology, vol. 26, no. 4, pp. 405-420, 1988.

[20] A. D. Barbour, "The asymptotic behaviour of birth and death and some related processes," Advances in Applied Probability, vol. 7, pp. 28-43, 1975.

[21] I. S. Gradshteyn and I. M. Ryzhik, Tables of Integrals, Series and Products, Academic Press, New York, NY, USA, 1980. 


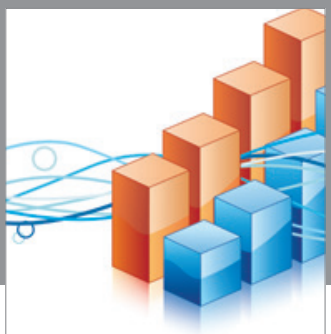

Advances in

Operations Research

vatem alat4

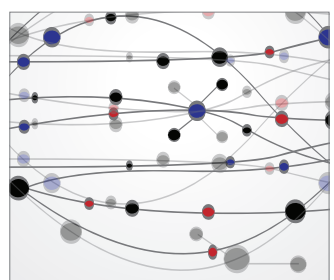

\section{The Scientific} World Journal
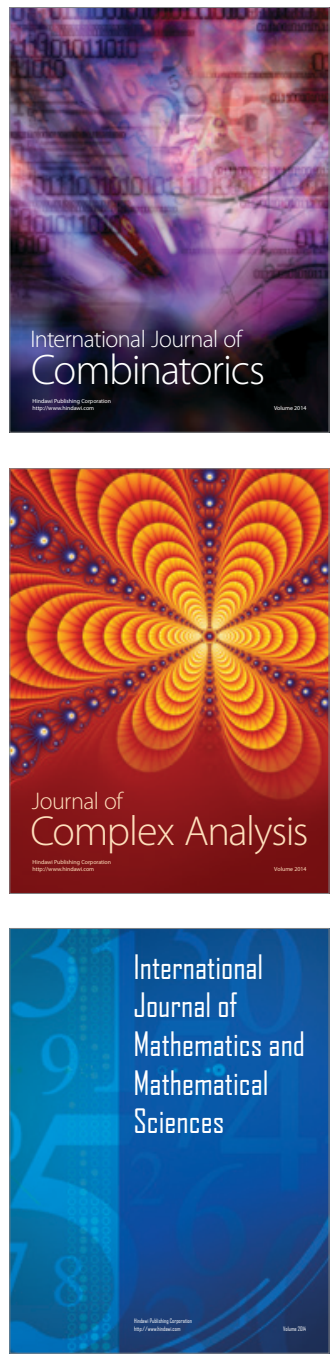
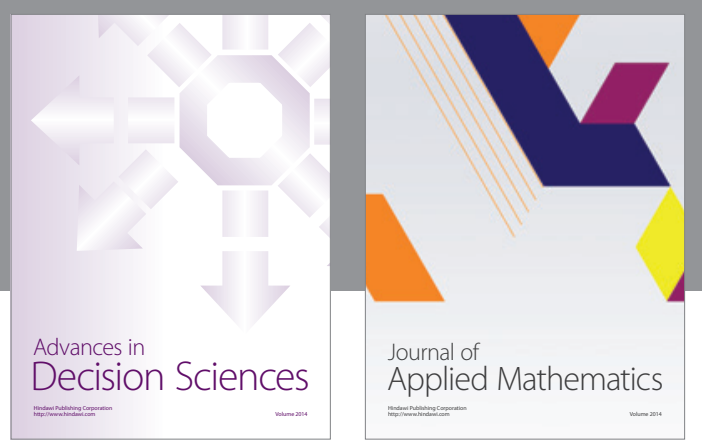

Algebra

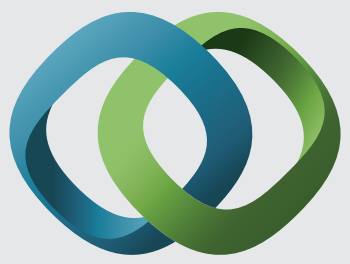

\section{Hindawi}

Submit your manuscripts at

https://www.hindawi.com
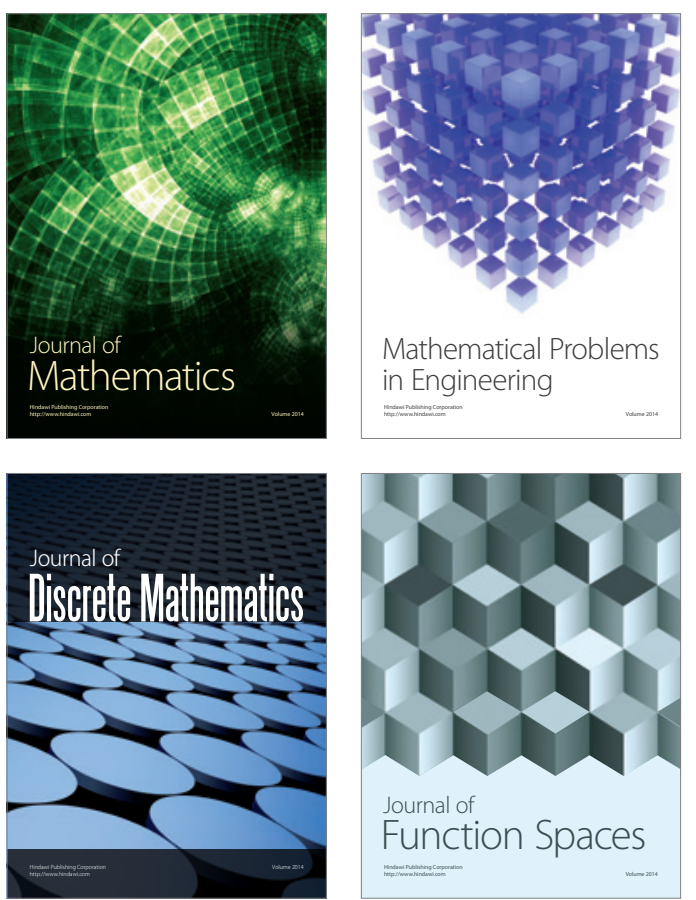

Mathematical Problems in Engineering
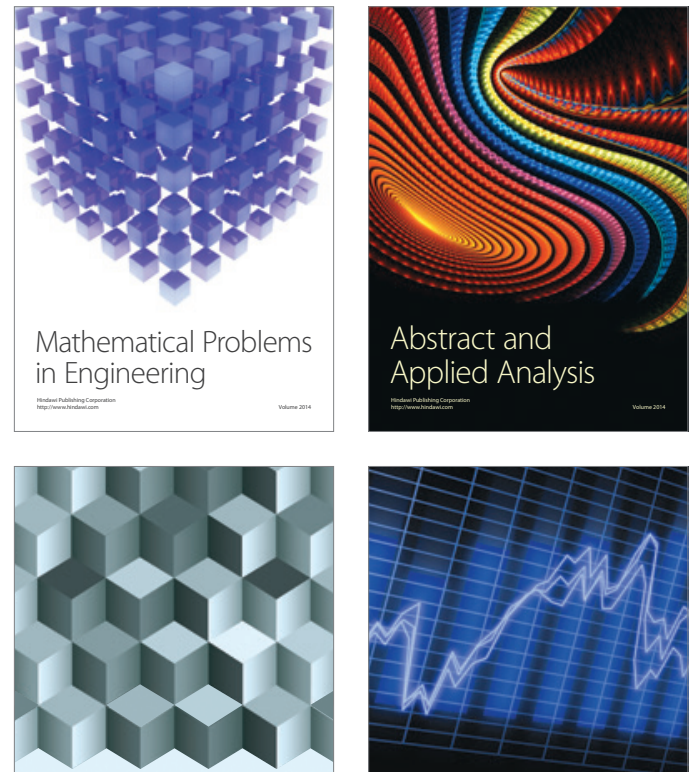

Journal of

Function Spaces

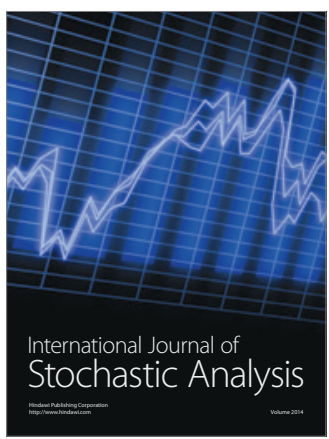

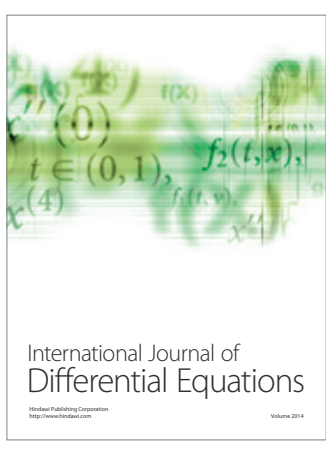
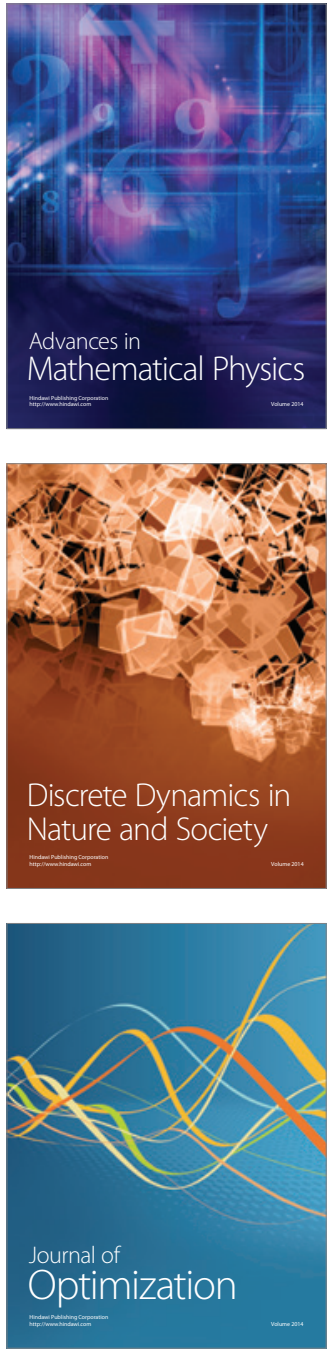